\title{
Renal resistive index and long-term outcome in patients with coronary artery disease
}

\author{
Maciej T. Wybraniec ${ }^{*}$ (D), Maria Bożentowicz-Wikarek², Magdalena Olszanecka-Glinianowicz'2 Jerzy Chudek ${ }^{2,3}$ and
} Katarzyna Mizia-Stec ${ }^{1}$

\begin{abstract}
Background: The study aimed to evaluate the application of intra-renal Doppler flow indices for the prediction of major adverse cardiac and cerebrovascular events (MACCE) during 24-month follow-up in patients with coronary artery disease (CAD) subject to coronary angiography (CA).

Methods: This prospective study comprised 111 consecutive patients with stable and unstable CAD (68.5\% men; median age 65 years), referred for CA. Ultrasonographic parameters of intra-renal blood flow in arcuate/interlobular arteries, including renal resistive index (RRI) and pulsatility index (RPI), were acquired directly before and $1 \mathrm{~h}$ after the procedure. Endpoint of MACCE (cardiovascular death, myocardial infarction, myocardial revascularization or stroke) were recorded during 24-month follow-up.

Results: MACCE occurred in 14 patients (12.6\%). Patients with MACCE had more diffuse CAD reflected by Syntax score (23.6 vs. 14.4 pts., $p=0.02)$, higher platelet level $(242.4$ vs. $207.2 \times 1000 / \mu l, p=0.01)$, higher rate of left main CAD ( $42.9 \%$ vs.5.2\%, $p<0.001)$ and left ventricular ejection fraction $<50 \%(50 \%$ vs.23.7\%, $p=0.045)$. Patients with MACCE had higher pre-procedural $(0.68 \pm 0.06$ vs. $0.62 \pm 0.06, p<0.001)$ and post-procedural RRI $(0.72 \pm 0.06$ vs.0.66 $\pm 0.06, p=0.01$ ), but comparable RPI ( $p=0.63$ and $p=0.36$, respectively). Cox proportional hazards model revealed that pre-procedural $\mathrm{RRI}(\mathrm{OR}=1.11$ per $0.01 ; p=0.02)$ and left main $C A D(O R=5.75, p=0.002)$ were the only independent predictors of MACCE occurrence. Receiver operator characteristic curve analysis revealed that preprocedural RRI $>0.645$ accurately predicted the composite endpoint (AUC $=0.78, p=0.001$ ) and identified patients with impaired 24-month prognosis according to Kaplan-Meier curve (log-rank $p<0.001)$.
\end{abstract}

Conclusions: Increased pre-procedural RRI, together with left main CAD, are associated with worse 24-month prognosis in patients with CAD referred for CA.

Keywords: Coronary artery disease, Renal resistive index, Renal pulsatility index, Major adverse cardiovascular events coronary artery disease

\footnotetext{
* Correspondence: maciejwybraniec@gmail.com

${ }^{1}$ First Department of Cardiology, School of Medicine in Katowice, Medical University of Silesia, 47 Ziołowa St., 40-635 Katowice, Poland

Full list of author information is available at the end of the article
}

(c) The Author(s). 2020 Open Access This article is licensed under a Creative Commons Attribution 4.0 International License, which permits use, sharing, adaptation, distribution and reproduction in any medium or format, as long as you give appropriate credit to the original author(s) and the source, provide a link to the Creative Commons licence, and indicate if changes were made. The images or other third party material in this article are included in the article's Creative Commons licence, unless indicated otherwise in a credit line to the material. If material is not included in the article's Creative Commons licence and your intended use is not permitted by statutory regulation or exceeds the permitted use, you will need to obtain permission directly from the copyright holder. To view a copy of this licence, visit http://creativecommons.org/licenses/by/4.0/. The Creative Commons Public Domain Dedication waiver (http://creativecommons.org/publicdomain/zero/1.0/) applies to the data made available in this article, unless otherwise stated in a credit line to the data. 


\section{Background}

In contrast to ST-segment elevation myocardial infarction, chronic coronary syndromes (CCS) and nonST-segment elevation acute coronary syndromes (NSTE-ACS) share different set of predictors of longterm outcome [1]. Numerous well-established clinical prognostic factors in NSTE-ACS patients exists, such as age, left ventricular ejection fraction (LVEF), completeness of revascularization, SYNTAX score [2], presence of acute heart failure, peak troponin elevation and ST-segment deviation [3]. Still, baseline renal function represents a central determinant of longterm mortality, reflecting the vital interplay between heart and kidneys [4]. The coexistence of cardiac and renal pathology, known as cardio-renal syndrome [5], is linked to humoral and neural signaling, and extends far beyond mere coincidence. The acute and chronic cardio-renal syndrome is partially modulated via catecholamine surge and increased sympathetic tone [5]. Although sympathetic nervous system hyperactivity has been deemed responsible for impaired survival [6], its evaluation is cumbersome and not amenable to routine clinical practice [7].

Renal vascular hemodynamics provides an indirect measure of sympathetic nervous function and can be easily assessed by means of Doppler ultrasound analysis of the spectrum of flow in interlobular and/or arcuate arteries [8]. Among numerous parameters, renal resistive index (RRI) was shown to be the most reproducible and clinically meaningful indices of both renal vascular resistance and stiffness $[9,10]$. RRI was shown to be dependent on numerous factors, including renal artery stiffness, age, pulse blood pressure, severe brady- and tachycardia, presence of valvular heart disease or any pathological lesions within renal parenchyma [11]. In daily clinical practice RRI may facilitate the diagnosis of renal artery stenosis [12] or help diagnose an acute rejection in kidney transplant recipients [13]. Assessment of pre-procedural RRI allowed for early identification of patients at risk of contrast-induced acute kidney injury (CI-AKI) among patients with coronary artery disease (CAD) referred for coronary angiography (CA) [14]. RRI also accurately predicted the onset [15] and persistence of acute kidney injury [16]. Moreover, RRI appears to be a general measure of arterial remodeling and stiffness and was linked to central pulse pressure values and echocardiographic parameters of left ventricular systolic and diastolic blood flow [10]. Accordingly, parameters of intra-renal blood flow were independently associated with occurrence of cardiovascular adverse events in an unselected elderly population [17] and RRI specifically, among patients with essential hypertension, especially in those with hypertensive renal disease [18]. Of note, in a large cohort of patients with chronic kidney disease
(CKD), elevated RRI was unequivocally associated with increased mortality [19]. Yet, none of the previously published studies evaluated RRI as a prognostic factor in patients with CAD undergoing cardiac catheterization.

The study aimed to verify the hypothesis that the use of intra-renal flow parameters may identify patients with coronary artery disease (CAD) at risk of major adverse cardiac and cerebrovascular events (MACCE) during 24month follow-up.

\section{Methods}

The study represents a prospective observation of different predictors of adverse cardiovascular events among patients with CAD referred to elective or urgent coronary angiography (CA), with special consideration of intra-renal Doppler flow parameters. The study covered consecutive one hundred and eleven patients with the clinical suspicion of CAD who met inclusion criteria of either: a) stable coronary syndrome with clinical indication for CA based on former non-invasive stress test or b) non-ST-segment elevation acute coronary syndrome (NSTE-ACS). The enrollment phase took place between 2014 and 2016.

The exclusion criteria involved cardiogenic shock, pulmonary edema, any sort of respiratory failure, chronic kidney disease $\left(\mathrm{eGFR}<50 \mathrm{ml} / \mathrm{min} / 1.73 \mathrm{~m}^{2}\right.$ or proteinuria $>500 \mathrm{mg} / \mathrm{l}$ ), renal structural abnormalities, active urinary tract infection, renal artery stenosis, pregnancy, previous hypersensitivity to contrast medium, morbid obesity (body mass index, BMI $\geq 40 \mathrm{~kg} / \mathrm{m}^{2}$ ). In addition, exclusion criteria entailed conditions that could alter the intra-renal hemodynamics, such as moderate to severe aortic valve stenosis, severe valvular heart disease of any kind, high pulse pressure $>80 \mathrm{mmHg}$, tachycardia $>100$ bpm or bradycardia $<50$ bpm.

The study was carried out in adherence to the principles of the Declaration of Helsinki and was formerly assessed and approved by local Ethics Committee. All study participants gave written informed consent to study participation.

The study protocol involved meticulous review of demographic and clinical data, as well as acquisition of blood specimen for rudimentary laboratory tests at baseline, including serum creatinine concentration (SCr). Furthermore, 24-h and 48-h blood samples were assayed for $\mathrm{SCr}$ in order to facilitate possible diagnosis of CI-AKI.

All the patients underwent renal ultrasound study with Doppler analysis of indices of blood flow within arcuate or interlobular arteries. The study was performed twice: directly before and $1 \mathrm{~h}$ following coronary angiography. The evaluation was conducted by a single experienced investigator by means of Vivid 7 (GE Healthcare) with a $5 \mathrm{C}$ probe $(4.4-6.7 \mathrm{MHz})$. Prior to the measurements, 
patients were required to remain supine for at least 10 min. First, the morphology of kidneys was assess in order to establish their length and width, as well as potential structural abnormalities precluding further evaluation. Subsequently, patients were screened for renal artery stenosis using peak systolic and end-diastolic flow velocity within the main renal artery. A $2-4 \mathrm{~mm}$ pulsewave Doppler gate was applied for intra-renal arteries in order to assess a set of indices, including peak systolic (PSV) and end-diastolic velocity (EDV), mean velocity $(\mathrm{MV})$, acceleration time (AT), augmentation index (AI). The above-mentioned parameters were then used to calculate renal resistive index (RRI) and renal pulsatility index (RPI) based on well-known formulas: RRI = (PSV$\mathrm{EDV}) / \mathrm{PSV}$ and RPI $=(\mathrm{PSV}-\mathrm{EDV}) / \mathrm{MV}$. The core indices were acquired 3 times on each side during both pre- and post-procedural evaluation. The final pre- and postprocedural values constituted arithmetic means of these measurements.

\section{Follow-up and primary endpoint}

Patients were followed up for 24-months using a structured telephone interview performed 1-month, 12-months and 24-months after the hospitalization. Furthermore, data on any available outpatient visit or recurrent hospital admission in the local electronic database was gathered.

The primary endpoint comprised all major adverse cerebral and cardiovascular events (MACCE), including cardiovascular death, myocardial infarction, need for urgent revascularization and ischemic stroke.

\section{Peri-procedural management}

All study participants were treated in line with the European Society of Cardiology 2014 Guidelines on Myocardial Revascularization [20]. The coronary angiography was performed using low- or iso-osmolar contrast agents. Although patients with CKD defined as eGFR < $50 \mathrm{ml} / \mathrm{min} / 1.73 \mathrm{~m}^{2}$ were excluded from the study, patients with borderline renal function (eGFR $50-60 \mathrm{ml} /$ min) obtained an intravenous drip of $0.9 \%$ saline $(1 \mathrm{ml} /$ $\mathrm{kg} / \mathrm{h} ; 12 \mathrm{~h}$ before -24 after coronary angiography). Patients with preserved kidney function (eGFR > $60 \mathrm{ml} /$ $\mathrm{min}$ ) received one $500 \mathrm{ml} 0.9 \%$ saline before the procedure. Biguanides were stopped $24 \mathrm{~h}$ prior to contrast.

\section{Definitions}

The CI-AKI was defined as $\geq 50 \%$ relative or $\geq 0.3 \mathrm{mg} / \mathrm{dL}$ absolute increase of $\mathrm{SCr}$ at $48 \mathrm{~h}$ after procedure [14]. Chronic kidney disease was diagnosed if eGFR was $<50$ $\mathrm{ml} / \mathrm{min} / 1.73 \mathrm{~m}^{2}$ or in case of documented proteinuria $>$ $500 \mathrm{mg} / \mathrm{l}$. Hypertension was defined if mean blood pressure from two measurements was $>140 / 90 \mathrm{mmHg}$ on two separate visits or antihypertensive agents regiment.
The severity of CAD was reflected by SYNTAX score, which was calculated using the official online calculator by an experienced invasive cardiologist.

\section{Statistical analysis}

Statistical calculations were processed using MedCalc v.14.8.1 software package (MedCalc, Ostend, Belgium). Continues variables were expressed as mean and standard deviation (SD) or median and 25-75 percentile boundaries, depending on the distribution, which was defined using Shapiro-Wilk's test. The difference between MACCE and non-MACCE cohort was established by means of Student's t test in case of normal distribution or Mann-Whitney $U$ in the instance of nonnormally distributed parameters. All the variables with $p<0.1$ in univariate model were incorporated into the Cox proportional hazards model. Hazard ratio (HR) with 95\% confidence intervals (95\% CI) were calculated. The diagnostic power of different intra-renal flow parameters was established using the receiver operating characteristics (ROC) curve analysis. Cut-off values of different flow parameters were defined on the basis of Youden's J statistic. Kaplan-Meier survival curves and log-rank tests were calculated. A $p$-value of less than 0.05 was deemed statistically significant across the analyses.

\section{Results \\ General characteristics}

The demographic and clinical characteristics of the population was highlighted in Table 1 . The study population comprised 111 patients referred to elective or urgent coronary angiography with the diagnosis of stable angina $(42.3 \%)$, or non-ST-elevation acute coronary syndrome $(57.7 \%)$. The population was characterized by the predominance of males $(68.5 \%)$. The population represented a typical cohort of CAD patients with median age of $65(59 ; 71)$ years and pronounced cardiovascular risk factors, such as high prevalence of cigarette smoking (59.5\%), hypertension (96.4\%), diabetes (37.8\%). Of note, history of past myocardial infarction in anamnesis was present in $45.9 \%$ of patients.

\section{Major adverse cerebral and cardiovascular events}

The total number of 14 MACCE events occurred in 14 patients (12.6\%). Three patients experienced acute myocardial infarction; eight patients underwent urgent percutaneous myocardial revascularization due to unstable angina and ischemic stroke occurred in three patients.

The comparison of MACCE and non-MACE cohorts was presented in Table 1. Patients who exhibited MACCE more often had left main CAD $(p<0.001)$, higher SYNTAX score $(p=0.02)$ and platelet count $(p=$ $0.01)$ and lower prevalence of depressed LVEF $(p=0.02)$. 
Table 1 Demographic and clinical characteristics of study population, stratified depending on the presence of MACCE during 24month follow-up

\begin{tabular}{|c|c|c|c|c|}
\hline Variable & $\begin{array}{l}\text { Whole population } \\
\boldsymbol{n}=111\end{array}$ & $\begin{array}{l}\text { Non-MACCE } \\
\boldsymbol{n}=97\end{array}$ & $\begin{array}{l}\text { MACCE } \\
\boldsymbol{n}=14\end{array}$ & $p$ \\
\hline Men & $76(68.5 \%)$ & $65(67.0 \%)$ & $11(78.6 \%)$ & $0.29^{\mathrm{a}}$ \\
\hline Age [years] & $65(59 ; 71)$ & $65(60 ; 71)$ & $63.5(57 ; 69)$ & $0.97^{b}$ \\
\hline BMI $\left[\mathrm{kg} / \mathrm{m}^{2}\right]$ & $29.1 \pm 4.5$ & $29.3 \pm 4.6$ & $27.8 \pm 3.0$ & $0.26^{c+}$ \\
\hline Waist circumference $[\mathrm{cm}]$ & $90.6 \pm 11.5$ & $91.0 \pm 11.9$ & $87.7 \pm 8.7$ & $0.32^{c}$ \\
\hline Cigarette smoking & $66(59.5 \%)$ & $57(58.8 \%)$ & 9 (64.3\%) & $0.47^{\mathrm{a}}$ \\
\hline Hypertension & $107(96.4 \%)$ & $93(95.9 \%)$ & $14(100.0 \%)$ & $0.58^{a}$ \\
\hline Diabetes type 2 & $42(37.8 \%)$ & $35(36.1 \%)$ & $7(50.0 \%)$ & $0.24^{a}$ \\
\hline Atrial fibrillation & $24(21.6 \%)$ & $23(23.7 \%)$ & $1(7.1 \%)$ & $0.14^{a}$ \\
\hline History of Ml & $51(45.9 \%)$ & $44(45.4 \%)$ & $7(50.0 \%)$ & $0.48^{a}$ \\
\hline History of ischemic stroke/TIA & $8(7.2 \%)$ & $8(8.3 \%)$ & $0(0.0 \%)$ & $0.26^{a}$ \\
\hline COPD & $9(8.1 \%)$ & $9(9.3 \%)$ & $0(0.0 \%)$ & $0.23^{a}$ \\
\hline Cl-AKI by AKIN criteria & $9(8.1 \%)$ & $6(6.2 \%)$ & $3(21.4 \%)$ & $0.09^{a}$ \\
\hline NSTE-ACS & $64(57.7 \%)$ & $54(55.7 \%)$ & $10(71.4 \%)$ & $0.27^{a}$ \\
\hline NSTEMI & $32(28.8 \%)$ & $27(27.8 \%)$ & $5(35.7 \%)$ & $0.37^{\mathrm{a}}$ \\
\hline Unstable angina & $32(28.8 \%)$ & $27(27.8 \%)$ & $5(35.7 \%)$ & $0.37^{a}$ \\
\hline Left main CAD & $11(9.9 \%)$ & $5(5.2 \%)$ & $6(42.9 \%)$ & $<0.001^{\mathrm{a}}$ \\
\hline SYNTAX score [pts] & $13(4 ; 25)$ & $12(3 ; 24)$ & $22.5(11 ; 36)$ & $0.02^{b}$ \\
\hline $\mathrm{PCl}$ ad hoc & $49(44.1 \%)$ & $42(43.3 \%)$ & $7(50.0 \%)$ & $0.64^{\mathrm{a}}$ \\
\hline CABG referral & $17(15.3 \%)$ & $13(13.4 \%)$ & $4(28.6 \%)$ & $0.14^{\mathrm{a}}$ \\
\hline Hemoglobin [g/dL] & $13.9 \pm 1.24$ & $14.0 \pm 1.22$ & $13.3 \pm 1.23$ & $0.07^{c}$ \\
\hline White blood cell count $\left[\times 1000 / \mathrm{mm}^{3}\right]$ & $7.07 \pm 1.73$ & $7.11 \pm 1.73$ & $6.74 \pm 1.78$ & $0.46^{c}$ \\
\hline Platelet count $\left[\times 1000 / \mathrm{mm}^{3}\right]$ & $201(177 ; 254)$ & $207.2 \pm 0.02$ & $242.4 \pm 0.02$ & $0.01^{c}$ \\
\hline Peak hsTnT $[\mathrm{ng} / \mathrm{mL}]$ & $0.013(0.008 ; 0.034)$ & $0.013(0.008 ; 0.032)$ & $0.023(0.012 ; 0.052)$ & $0.46^{b}$ \\
\hline Serum creatinine $[\mathrm{mg} / \mathrm{dL}]$ & $0.92(0.79 ; 1.13)$ & $0.92(0.79 ; 1.11)$ & $1.03(0.83 ; 1.25)$ & $0.23^{b}$ \\
\hline eGFR $\left[\mathrm{ml} / \mathrm{min} / 1.73 \mathrm{~m}^{2}\right]$ & $80.3 \pm 20.8$ & $80.5 \pm 20.6$ & $79.2 \pm 23.4$ & $0.83^{c}$ \\
\hline $\mathrm{IMT}[\mathrm{mm}]$ & $0.10 \pm 0.03$ & $0.09 \pm 0.03$ & $0.10 \pm 0.02$ & $0.17^{c}$ \\
\hline LVEF [\%] & $53.3 \pm 7.3$ & $53.2 \pm 7.2$ & $53.3 \pm 7.7$ & $0.99^{c}$ \\
\hline $\mathrm{LVEF}<50 \%$ & $30(27.0 \%)$ & $23(23.7 \%)$ & $7(50.0 \%)$ & $0.045^{\mathrm{a}}$ \\
\hline$E / e^{\prime}$ & $8.6(6.7 ; 12.0)$ & $8.6(6.7 ; 12.1)$ & $6.8(6.4 ; 10.0)$ & $0.06^{b}$ \\
\hline Mitral valve insufficiency & $72(64.9 \%)$ & $66(68.0 \%)$ & $6(42.9 \%)$ & $0.07^{a}$ \\
\hline Systolic BP [mmHg] & $130(120 ; 140)$ & $130(120 ; 140)$ & $140(120 ; 150)$ & $0.33^{b}$ \\
\hline Diastolic BP [mmHg] & $80(70 ; 90)$ & $80(70 ; 90)$ & $80(70 ; 100)$ & $0.15^{b}$ \\
\hline Pulse BP [mmHg] & $40(30 ; 50)$ & $40(30 ; 50)$ & $40(30 ; 55)$ & $0.79^{a}$ \\
\hline Vmax - abdominal aorta $[\mathrm{m} / \mathrm{s}]$ & $0.60 \pm 0.13$ & $0.59 \pm 0.13$ & $0.61 \pm 0.17$ & $0.73^{c}$ \\
\hline RRI pre. & $0.63 \pm 0.07$ & $0.62 \pm 0.06$ & $0.68 \pm 0.06$ & $<0.001^{c}$ \\
\hline RRI post. & $0.68 \pm 0.07$ & $0.66 \pm 0.06$ & $0.72 \pm 0.06$ & $0.01^{c}$ \\
\hline$\Delta R R I$ & $0.05 \pm 0.05$ & $0.05 \pm 0.05$ & $0.04 \pm 0.04$ & $0.42^{c}$ \\
\hline RPI pre. & $1.40(1.25 ; 1.52)$ & $1.39 \pm 0.20$ & $1.36 \pm 0.25$ & $0.63^{c}$ \\
\hline RPI post. & $1.51(1.36 ; 1.64)$ & $1.48 \pm 0.21$ & $1.54 \pm 0.24$ & $0.36^{c}$ \\
\hline$\triangle \mathrm{RPI}$ & $0.10 \pm 0.19$ & $0.09 \pm 0.20$ & $0.18 \pm 0.13$ & $0.11^{c}$ \\
\hline PSV pre. $[\mathrm{m} / \mathrm{s}]$ & $0.42 \pm 0.09$ & $0.43 \pm 0.09$ & $0.40 \pm 0.11$ & $0.33^{c}$ \\
\hline PSV post. [m/s] & $0.45 \pm 0.08$ & $0.45 \pm 0.08$ & $0.45 \pm 0.10$ & $0.83^{c}$ \\
\hline EDV pre. $[\mathrm{m} / \mathrm{s}]$ & $0.16 \pm 0.05$ & $0.16 \pm 0.05$ & $0.13 \pm 0.05$ & $0.01^{c}$ \\
\hline
\end{tabular}


Table 1 Demographic and clinical characteristics of study population, stratified depending on the presence of MACCE during 24month follow-up (Continued)

\begin{tabular}{|c|c|c|c|c|}
\hline Variable & $\begin{array}{l}\text { Whole population } \\
\boldsymbol{n}=111\end{array}$ & $\begin{array}{l}\text { Non-MACCE } \\
\boldsymbol{n}=97\end{array}$ & $\begin{array}{l}\text { MACCE } \\
\boldsymbol{n}=14\end{array}$ & $p$ \\
\hline EDV post. [m/s] & $0.15 \pm 0.05$ & $0.15 \pm 0.05$ & $0.12 \pm 0.04$ & $0.045^{c}$ \\
\hline AcT pre. [ms] & $61(51 ; 69.5)$ & $57(50.5 ; 68.5)$ & $69.75(58.5 ; 77.5)$ & $0.06^{b}$ \\
\hline AcT post. [ms] & $82(68.5 ; 98.0)$ & $77.5(65.5 ; 98.0)$ & $94.0(85.5 ; 107.0)$ & $0.11^{b}$ \\
\hline$\triangle \mathrm{AcT}[\mathrm{ms}]$ & $18.5(9.5 ; 32.5)$ & $18.5(9.5 ; 31.0)$ & $19.0(17.5 ; 38.0)$ & $0.66^{b}$ \\
\hline Al pre. $\left[\mathrm{m} / \mathrm{s}^{2}\right]$ & $4.1(3.6 ; 4.6)$ & $4.1(3.7 ; 4.6)$ & $3.7(3.4 ; 4.2)$ & $0.42^{b}$ \\
\hline Al post. $\left[\mathrm{m} / \mathrm{s}^{2}\right]$ & $3.6(3.1 ; 4.1)$ & $3.5(3.2 ; 4.0)$ & $3.6(3.0 ; 4.3)$ & $0.90^{b}$ \\
\hline$\Delta \mathrm{Al}\left[\mathrm{m} / \mathrm{s}^{2}\right]$ & $-0.5(0.9 ; 0.1)$ & $-0.5(-0.9 ;-0.1)$ & $-0.5(-0.9 ;-0.1)$ & $0.36^{b}$ \\
\hline
\end{tabular}

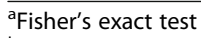

${ }^{b}$ Mann-Whitney's U test

'Student's t test

AKIN Acute Kidney Injury Network criteria, BMI Body mass index, COPD Chronic obstructive pulmonary disease, LVEF Left ventricular ejection fraction, MVI Mitral valve insufficiency, LM Left main, RRI Renal resistive index, RPI Renal pulsatility index, Al Acceleration index, AcT Acceleration time, PSV Peak systolic velocity, EDV End-diastolic velocity, NSTE-ACS Non-ST-segment elevation acute coronary syndrome, NSTEMI Non-ST-elevation myocardial infarction, Cl-AKI Contrast-induced acute kidney injury, PCI Percutaneous coronary intervention, pre. Pre-procedural, post. Post-procedural, LVEF Left ventricular ejection fraction, hs-TnT High sensitivity troponin $\mathrm{T}$, eGFR Estimated glomerular filtration rate

Both groups did not differ in terms of rate of myocardial infarction or ad hoc PCI.

Most importantly, MACCE cohort had higher pre$(0.68 \pm 0.06$ vs. $0.62 \pm 0.06, \quad p<0.001)$ and postprocedural RRI $(0.72 \pm 0.06$ vs. $0.66 \pm 0.06, p=0.01)$ and lower intra-renal pre- $(0.13 \pm 0.05$ vs. $0.16 \pm 0.05 \mathrm{~m} / \mathrm{s}$, $p=0.01)$ and post-procedural EDV $(0.12 \pm 0.04$ vs. $0.15 \pm 0.05 \mathrm{~m} / \mathrm{s}, p=0.045)$. Other intra-renal Doppler flow parameters did not differ between study groups (Table 1).

\section{ROC analysis and Kaplan-Meier survival curves}

The ROC curve analysis was showed in Table 2 . The analysis revealed that both pre- (AUC $0.780 ; p<0.001$; cut-off $>0.645$; Fig. 1 ) and post-procedural (AUC 0.715; $p=0.003$; cut-off $>0.699$; Fig. 1$)$ RRI had a good diagnostic power in prediction of MACCE. These threshold values of RRI were subsequently applied for KaplanMeier survival curves. Patients characterized by preprocedural RRI $>0.645$ had significantly higher risk of MACCE as reflected by Kaplan-Meier survival curve (log-rank $p<0.001$; Fig. 2), as well as patients with postprocedural RRI $>0.699$ (log-rank $p=0.004)$. Patients with pre-procedural intra-renal EDV $<0.13 \mathrm{~m} / \mathrm{s}$ had also more favorable outcome (log-rank $p=0.006$ ).

Also, the presence of left main CAD (log-rank $p<$ $0.0001)$, but neither the diagnosis of NSTEMI $(p=0.52)$, nor PCI at index hospitalization $(p=0.67)$ stratified the population in terms of survival free from MACCE.

\section{Cox proportional hazards model}

The results of univariate and multivariate Cox regression analysis are presented in Table 3 . After inclusion of all statistically significant (or borderline; $p<0.1$ ) variables, the final model yielded pre-procedural RRI (OR 1.11 per
0.01, 95\%CI: $1.02-1.20, p=0.02)$ and left main CAD (OR 5.75, 95\%CI: $1.88-17.62, p=0.002$ ) as significant predictors of MACCE during 24-month follow-up.

\section{Discussion}

The present study delivered evidence for significantly higher values of both pre- and post-procedural RRI in

Table 2 Receiver operating characteristics curve analysis of different laboratory predictors of 12-month MACCE occurrence

\begin{tabular}{|c|c|c|c|c|c|c|}
\hline \multicolumn{2}{|c|}{ Variable } & \multirow{2}{*}{$\begin{array}{l}\text { Criterion } \\
>0.645\end{array}$} & \multirow{2}{*}{$\begin{array}{l}\text { Sensitivity } \\
85.7\end{array}$} & \multirow{2}{*}{$\begin{array}{l}\text { Specificity } \\
63.9\end{array}$} & \multirow{2}{*}{$\frac{A U C}{0.780}$} & \multirow{2}{*}{$\begin{array}{l}p \\
<0.001\end{array}$} \\
\hline$\overline{\mathrm{RRI}}$ & Baseline & & & & & \\
\hline & Post-proc. at $1 \mathrm{~h}$ & $>0.699$ & 78.6 & 62.9 & 0.715 & 0.003 \\
\hline & $\Delta$ & $\leq 0.049$ & 78.6 & 46.4 & 0.570 & 0.36 \\
\hline \multirow[t]{3}{*}{ RPI } & Baseline & $\leq 1.185$ & 35.7 & 84.5 & 0.500 & 0.99 \\
\hline & Post-proc. at $1 \mathrm{~h}$ & $>1.595$ & 50.0 & 73.2 & 0.584 & 0.38 \\
\hline & $\Delta$ & $>0.2$ & 50.0 & 77.3 & 0.635 & 0.09 \\
\hline \multirow[t]{3}{*}{$\mathrm{Al}$} & Baseline & $\leq 3.80$ & 57.1 & 73.2 & 0.616 & 0.18 \\
\hline & Post-proc. at $1 \mathrm{~h}$ & $\leq 3.13$ & 42.9 & 80.4 & 0.522 & 0.80 \\
\hline & $\Delta$ & $>0.74$ & 14.3 & 100.0 & 0.523 & 0.79 \\
\hline \multirow[t]{3}{*}{ AT } & Baseline & $>71.5$ & 50.0 & 83.5 & 0.663 & 0.06 \\
\hline & Post-proc. at $1 \mathrm{~h}$ & $>82$ & 85.7 & 55.7 & 0.684 & 0.004 \\
\hline & $\Delta$ & $>10$ & 100.0 & 29.9 & 0.593 & 0.17 \\
\hline \multirow[t]{3}{*}{ PSV } & Baseline & $\leq 0.27$ & 21.4 & 95.9 & 0.542 & 0.64 \\
\hline & Post-proc.at $1 \mathrm{~h}$ & $\leq 0.46$ & 64.3 & 51.6 & 0.520 & 0.83 \\
\hline & $\Delta$ & $>0.06$ & 28.6 & 86.6 & 0.559 & 0.49 \\
\hline \multirow[t]{3}{*}{ EDV } & Baseline & $\leq 0.13$ & 64.3 & 72.2 & 0.706 & 0.01 \\
\hline & Post-proc.at $1 \mathrm{~h}$ & $\leq 0.13$ & 71.4 & 62.9 & 0.679 & 0.02 \\
\hline & $\Delta$ & $>-0.02$ & 42.9 & 41.2 & 0.503 & 0.98 \\
\hline
\end{tabular}

Al Augmentation index, AUC Area under curve, CAD Coronary artery disease, Cl-AKI Contrast-induced acute kidney injury, HR Hazard ratio, Cl Confidence interval, RPI Renal pulsatility index, RRI Renal resistive index, PSV Peak systolic velocity, EDV End-diastolic velocity, AT Acceleration time 

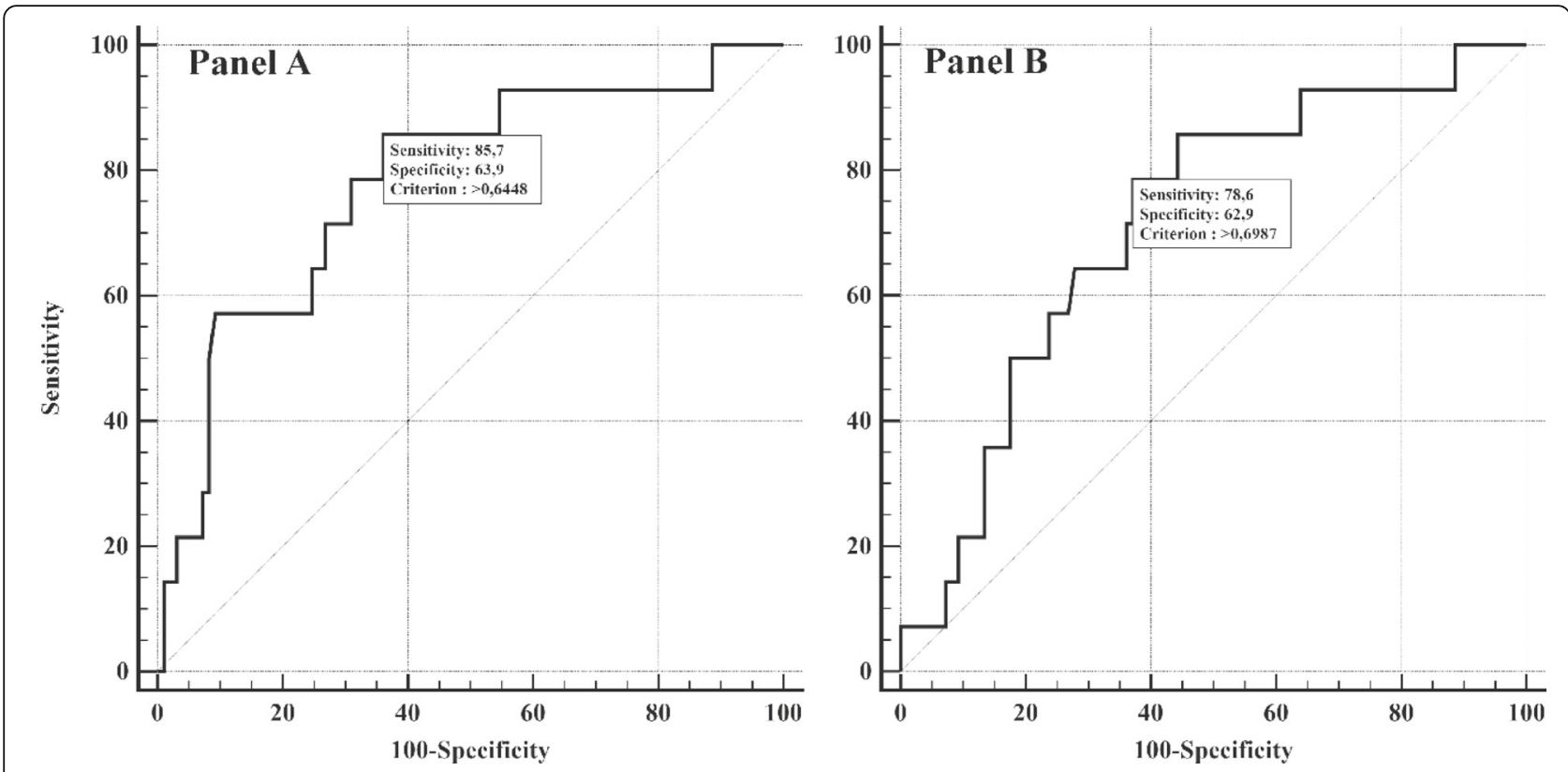

Panel A - pre-procedural renal resistive index

\section{Panel B - post-procedural renal resistive index}

Fig. 1 Receiver operating characteristics curve showing pre- and post-procedural renal resistive index as a predictor of major adverse cardiovascular events. $\mathbf{a}$ - pre-procedural renal resistive index. $\mathbf{b}$ - post-procedural renal resistive index

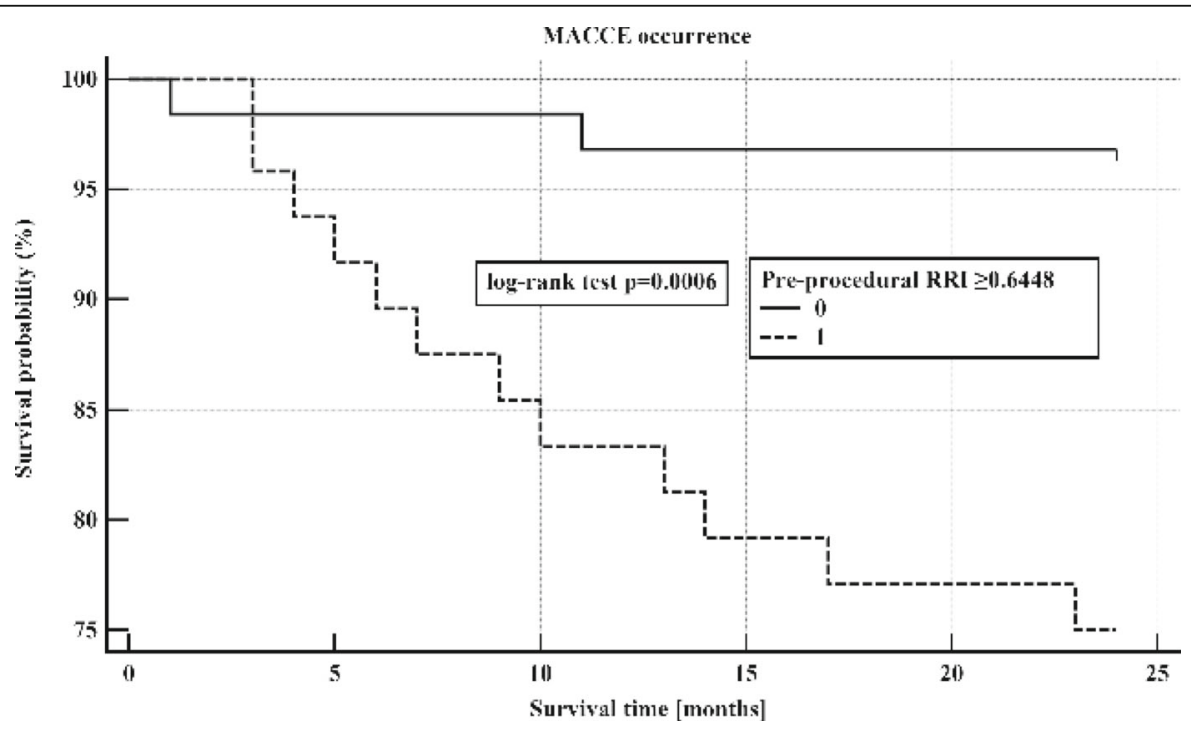

MACCE - major adverse cerebral and cardiovascular events; RRI - renal resistive index

Fig. 2 Kaplan-Meier survival curve of MACCE occurrence during 24-month observation depending on the level of pre-procedural RRI: $\geq$ or $<$ the jstatistic threshold of 0.6448. MACCE - major adverse cerebral and cardiovascular events; RRI - renal resistive index 
Table 3 Univariate and multivariate Cox proportional hazards model of different predictors of MACCE onset during 24-monh follow-

\begin{tabular}{|c|c|c|c|c|c|c|}
\hline \multirow[t]{2}{*}{ Variable } & \multicolumn{3}{|c|}{ Univariate analysis } & \multicolumn{3}{|c|}{$\begin{array}{l}\text { Stepwise multivariate Cox regression } \\
\text { Overall model fit: } \boldsymbol{p}=0.0001 \\
\text { Chi-square }=18.668\end{array}$} \\
\hline & $\overline{\mathrm{HR}}$ & $95 \% \mathrm{Cl}$ & $p$ & $\overline{H R}$ & $95 \% \mathrm{Cl}$ & $p$ \\
\hline Male sex & 1.77 & $0.50-6.30$ & 0.38 & - & - & - \\
\hline Age [per 1 years] $]^{a}$ & 0.99 & $0.94-1.06$ & 0.96 & - & - & - \\
\hline Arterial hypertension & 21.31 & $<0.001->1000$ & 0.62 & - & - & - \\
\hline Diabetes mellitus & 1.68 & $0.59-4.78$ & 0.33 & - & - & - \\
\hline Smoking & 1.28 & $0.43-3.79$ & 0.66 & - & - & - \\
\hline Cl-AKI onset & 3.56 & $1.00-12.73$ & 0.05 & - & - & - \\
\hline Left main CAD & 9.00 & $3.11-26.03$ & $<0.001$ & 5.75 & $1.88-17.62$ & 0.002 \\
\hline Syntax score [per 1 point] $]^{a}$ & 1.04 & $1.01-1.08$ & 0.02 & - & - & - \\
\hline Platelet count $\left[\text { per } 1 / \mathrm{mm}^{3}\right]^{a}$ & 1.01 & $1.00-1.02$ & 0.02 & - & - & - \\
\hline RRI pre. [per 0.01] $]^{\mathrm{a}}$ & 1.14 & $1.05-1.22$ & 0.001 & 1.11 & $1.02-1.20$ & 0.02 \\
\hline RRI post. [per 0.01] ${ }^{a}$ & 1.11 & $1.02-1.21$ & 0.02 & - & - & - \\
\hline EDV pre. [per $0.01 \mathrm{~m} / \mathrm{s}]^{\mathrm{a}}$ & 0.86 & $0.76-0.96$ & 0.01 & & - & - \\
\hline EDV post. [per $0.01 \mathrm{~m} / \mathrm{s}]^{\mathrm{a}}$ & 0.87 & $0.75-0.99$ & 0.04 & - & - & - \\
\hline AT pre. [per $1 \mathrm{~ms}]^{\mathrm{a}}$ & 1.02 & $1.00-1.04$ & 0.06 & - & - & - \\
\hline
\end{tabular}

anit odds ratio

CAD Coronary artery disease, CI-AKI Contrast-induced acute kidney injury, HR Hazard ratio, Cl Confidence interval, RRI Renal resistive index, EDV End-diastolic velocity, AT Acceleration time

patients with MACCE than in patients with uneventful 24-month follow-up after coronary angiography (Table 1). The difference in RRI was predominantly triggered by markedly lower pre- and post-procedural EDV (Table 1). Cox proportional hazards model corroborated that pre-procedural RRI and presence of LM disease were independent predictors of MACCE at 24-month follow-up (Table 3). Patients with pre-procedural RRI above the threshold of 0.645 had significantly worse MACCE-free survival than patients with low levels of RRI according to Kaplan-Meier survival curve (Fig. 2).

These findings for the first time shed light on the prognostic aspect of Doppler-derived RRI, mediated by EDV as its crucial determinant, in patients with atherosclerotic cardiovascular disease, since it accurately identified patients at risk of future adverse cardiovascular events. In the study by Pearce and coworkers performed on a large cohort of 870 elderly patients (mean age of $77 \pm 5$ years) participating in Cardiovascular Health Study [17], end-diastolic velocity was inversely associated with the onset of composite endpoint of fatal or nonfatal myocardial infarction, hospitalization for angina, need for PCI or CABG, fatal or nonfatal stroke or transient ischemic attack (HR: 0.73, 95\%CI: 0.62-0.87, $p<0.001$ ). The RRI itself expressed as a qualitative variable (RRI $\geq$ or $<0.8$ ) failed to predict onset of MACCE (HR 1.10, 95\%CI: $0.81-1.50, p=0.5)$. Still, RRI is dependent on EDV value and presumably RRI should be treated as a continuous variable, as opposed to using an arbitrary high threshold of 0.8 . In our study, pre-procedural RRI values as low as 0.645 stratified the population in terms of long-term outcome (Fig. 2).

The report by Doi et al. delivered proof that RRI is predictive of adverse cardiovascular events in a broad group of patients with arterial hypertension [18]. This study covered 426 patients without cardiovascular disorders in anamnesis and found that RRI was associated with MACCE occurrence (HR 1.81 for 1 SD increase, 95\%CI: $1.45-2.27, p<0.01$ ) [18]. The prognosis was worse among patients with RRI $\geq 0.65$ for males and 0.68 for females $(\log$ rank $p<0.01)[18]$. Contrary to the cited results, our study enrolled patients with symptomatic CAD, however, the vast majority of patients (96.4\%) also had hypertension. Unlike our research, which used CKD as an exclusion criterion, the study by Doi enrolled 133 patients with eGFR $<60 \mathrm{ml} / \mathrm{min} / 1.73 \mathrm{~m}^{2}$, precluding direct comparison of its results [18]. It is vital to note that RRI exhibited higher predictive power among patients with CKD as opposed to patients with preserved renal function (HR 2.11 for 1 SD increase, 95\%CI: 1.44-3.16, $p<0.01)$.

The predicate role of RRI in patients with impaired renal function and arterial hypertension was confirmed in the study Toledo et al. Based on a cohort of 1962 patients with CKD (eGFR $15-59 \mathrm{ml} / \mathrm{min}$ ), the authors provided evidence that RRI $>0.70$ was associated with higher long-term mortality (HR 1.29, 95\%CI: 1.02-1.65, $p<0.05)$, especially in younger group and in patients 
with stage 3 CKD [19]. This study was the only trial, which unequivocally linked RRI to mortality [19], not only to composite endpoint.

The results of the current research are novel as the study exclusively covered patients with preserved renal function (eGFR $\geq 50 \mathrm{ml} / \mathrm{min} / 1.73 \mathrm{~m}^{2}$ ), hence it highlights the clinical benefits of RRI assessment regardless of baseline kidney condition, which could alter the results of RRI.

The mechanism underlying the predictive role is presumably related to its association with structural vascular remodeling (stiffness) and sympathetic tone (renal artery constriction). The current study design is unique as it compensated for the possible variations of intrarenal flow parameters by excluding patients with extreme values of heart rate, pulse blood pressure or valvular heart disease. All these parameters remain significant determinants of RRI and also long-term outcome. Of note, pulse blood pressure represents a strong predictor of cardiovascular outcome and RRI is highly dependent on its value [10]. In our study, pulse blood pressure was comparable between both groups, yet RRI was not and thus other mechanisms drove higher RRI values in patients at risk of future adverse cardiovascular events. Presumably higher sympathetic tone within renal arcuate and interlobular arteries might contribute to lower EDV and higher RRI values in high risk patients. It is meaningful that pre-procedural values, without the deleterious impact of contrast media, had the highest predictive value towards long-term outcome.

These results correspond with former studies in the field, which showed association of higher level of cardiovascular risk factors among patients with higher RRI values [9, 10, 21]. Prejbisz et al. revealed that patients with truly resistant hypertension had higher RRI values than patients with well-controlled arterial hypertension $(0.62 \pm 0.05$ vs. $0.60 \pm$ $0.05, p<0.05)$ [21]. RRI exhibited correlation with ambulatory blood pressure, pulse blood pressure, fasting glucose concentration and E/e' ratio [21]. The metabolic aspect of RRI was documented on the basis of patients with type 2 diabetes, in whom dynamic RRI (change of RRI following administration of sublingual nitrate administration) predicted onset of microalbuminuria [22].

Last but not least, Geraci et al. delineated that RRI values were associated with the extent of carotid atherosclerosis [23]. In their study RRI correlated with carotid IMT assessed in patients with hypertension, both with $(r=0.42)$ and without CKD $(r=0.39)$ [23]. More recently, the same group of authors provided evidence that Doppler-derived intra-renal flow parameters were associated with the severity of coronary atherosclerosis assessed in coronary angiography, but only among patients with less pronounced atherosclerosis [24]. Of note, this association was valid for RPI, but not RRI, which requires further explanation [24].
In the former study conducted in our institution, RRI was documented to be an independent predictor of CIAKI [14]. Although CI-AKI rate was higher in MACCE cohort in the present analysis, it did not reach statistical significance and impaired prognosis cannot be solely attributed to this covariate [14]. Conversely, the presence of LM disease was a powerful predictor of event-free survival (HR 5.75, 95\%CI: 1.88-17.62, $p=0.002$ ), which corresponds with general risk stratification demonstrated in large clinical trials.

\section{Study limitations}

The main disadvantage of the study is limitation of RRI itself, as it depends on numerous variables. Still, subjects with extreme values of possible determinants of RRI [25], such as elderly age, severe tachy- and bradycardia, high pulse pressure were excluded from the study, which prevented hypothetical impact of these variables on study results. Also, the measurement of PSV and EDV and its derivatives is limited by rather high inter- and intra-observer variability. We attempted at limiting this shortcoming by conducting repeated measurement in both kidneys and using an arithmetic mean of all measurements.

\section{Conclusions}

Increased pre-procedural renal resistive index, together with left main CAD, are linked to 24-month prognosis in patients with CAD referred for coronary catheterization. This easily accessible diagnostic tool could improve the stratification of cardiovascular risk in CAD patients referred for invasive procedures.

\section{Abbreviations}

AKIN: Acute kidney injury network criteria; AcT: Acceleration time; Al: Acceleration index; AUC: Area under curve; BMI: Body mass index; CA: Coronary angiography; CABG: Coronary artery bypass grafting; CAD: Coronary artery disease; Cl: Confidence interval; CI-AKI: Contrastinduced acute kidney injury; CKD: Chronic kidney disease; COPD: Chronic obstructive pulmonary disease; eGFR: Estimated glomerular filtration rate; HR: Hazard ratio; LM: Left main; LVEF: Left ventricular ejection fraction; MACCE: Major adverse cardiac and cerebrovascular events; MVI: Mitral valve insufficiency; NSTE-ACS: Non-ST-segment elevation acute coronary syndrome; NSTEMI: Non-ST-elevation myocardial infarction; PCI: Percutaneous coronary intervention; ROC: Receive operating characteristics curve; RRI: Renal resistive index; RPI: Renal pulsatility index; PSV: Peak systolic velocity; EDV: Enddiastolic velocity; pre: Pre-procedural; post: Post-procedural; hs-TnT: High sensitivity troponin T; SCr: Serum creatinine concentration; SD: Standard deviation

\section{Acknowledgements}

Not applicable.

\section{Authors' contributions}

All authors have read and approved the manuscript. MTW - conceived the concept of the study, drafted the manuscript, performed ultrasonographic examination, collected baseline and follow-up data, performed statistical analysis; MBW - performed laboratory tests; drafted the manuscript; MOG reviewed the article; drafted the manuscript; JCH - reviewed the article; performed laboratory tests; drafted study manuscript; KMS - conceived the concept of the study; drafted study manuscript. 


\section{Funding}

The study was financed from the grant for scientific research in the field of cardio-nephrology funded by Adamed Group under the auspices of Polish Society of Cardiology. The funds enabled for performing the ultrasonographic examination and laboratory tests, as well as urinary analysis of patients subject the coronary angiography. The funder had no impact on the course of the project. The funds were assigned by an unbiased scientific committee following a meticulous assessment of the project.

\section{Availability of data and materials}

Upon notice, the authors are willing to share their raw data with all the interested parties.

\section{Ethics approval and consent to participate}

The study was carried out in adherence to the principles of the Declaration of Helsinki and was formerly assessed and approved by Ethics Committee of Medical University of Silesia in Katowice. All study participants gave written informed consent to study participation.

\section{Consent for publication}

All the authors give their consent for publication of the results.

\section{Competing interests}

The authors deny any potential conflict of interests regarding the contents of the article.

\section{Author details}

${ }^{1}$ First Department of Cardiology, School of Medicine in Katowice, Medical University of Silesia, 47 Ziołowa St., 40-635 Katowice, Poland. ${ }^{2}$ Department of Pathophysiology, School of Medicine in Katowice, Medical University of Silesia, Katowice, Poland. ${ }^{3}$ Department of Internal Medicine and Oncological Chemotherapy, Medical University of Silesia, Katowice, Poland.

Received: 26 November 2019 Accepted: 29 June 2020

Published online: 06 July 2020

\section{References}

1. Roffi M, Patrono C, Collet JP, Mueller C, Valgimigli M, Andreotti F, Bax JJ, Borger MA, Brotons C, Chew DP, Gencer B, Hasenfuss G, Kjeldsen K, Lancellotti P, Landmesser U, Mehilli J, Mukherjee D, Storey RF, Windecker S, ESC Scientific Document Group. 2015 ESC guidelines for the management of acute coronary syndromes in patients presenting without persistent STsegment elevation: Task Force for the Management of Acute Coronary Syndromes in patients presenting without persistent ST-segment elevation of the European Society of Cardiology (ESC). Eur Heart J. 2016;37:267-315.

2. Kappetein AP, Dawkins KD, Mohr FW, Morice MC, Mack MJ, Russell ME, Pomar J, Serruys PW. Current percutaneous coronary intervention and coronary artery bypass grafting practices for three-vessel and left main coronary artery disease. Insights from the SYNTAX run-in phase. Eur J Cardiothorac Surg. 2006;29:486-91.

3. de Araújo GP, Ferreira J, Aguiar C, Seabra-Gomes R. TIMI, PURSUIT, and GRACE risk scores: sustained prognostic value and interaction with revascularization in NSTE-ACS. Eur Heart J. 2005;26:865-72.

4. Zhang DQ, Li HW, Chen HP, Ma Q, Chen H, Xing YL, Zhao XQ. Combination of Amino-Terminal Pro- BNP, Estimated GFR, and High-Sensitivity CRP for Predicting Cardiorenal Syndrome Type 1 in Acute Myocardial Infarction Patients. J Am Heart Assoc. 2018;7:e009162.

5. Rangaswami J, Bhalla V, Blair JEA, Chang TI, Costa S, Lentine KL, Lerma EV, Mezue K, Molitch M, Mullens W, Ronco C, Tang WHW, McCullough PA. American Heart Association Council on the kidney in cardiovascular disease and council on clinical cardiology. Cardiorenal syndrome: classification, pathophysiology, diagnosis, and treatment strategies: A scientific statement from the American Heart Association. Circulation. 2019;139:e840-78.

6. Rizas KD, McNitt S, Hamm W, Massberg S, Kääb S, Zareba W, Couderc JP, Bauer A. Prediction of sudden and non-sudden cardiac death in postinfarction patients with reduced left ventricular ejection fraction by periodic repolarization dynamics: MADIT-II substudy. Eur Heart J. 2017;38:2110-8.

7. Ajijola OA, Lux RL, Khahera A, Kwon O, Aliotta E, Ennis DB, Fishbein MC, Ardell JL, Shivkumar K. Sympathetic modulation of electrical activation in normal and infarcted myocardium: implications for arrhythmogenesis. Am J Physiol Heart Circ Physiol. 2017;312:H608-21.
8. Di Nicolò P, Granata A. Renal resistive index: not only kidney. Clin Exp Nephrol. 2017;21:359-66.

9. Calabia J, Torguet P, Garcia I, Martin N, Mate G, Marin A, Molina C, Valles M. The relationship between renal resistive index, arterial stiffness, and atherosclerotic burden: the link between macrocirculation and microcirculation. J Clin Hypertens (Greenwich). 2014;16:186-91.

10. Kuznetsova T, Cauwenberghs N, Knez J, Thijs L, Liu YP, Gu YM, Staessen JA. Doppler indexes of left ventricular systolic and diastolic flow and central pulse pressure in relation to renal resistive index. Am J Hypertens. 2015;28:535-45.

11. Lerolle N. Please don't call me RI anymore; I may not be the one you think I am! Crit Care. 2012;16:174.

12. Boddi M. Renal ultrasound (and Doppler Sonography) in hypertension: an update. Adv Exp Med Biol. 2017;956:191-208.

13. Naesens M, Heylen L, Lerut E, Claes K, De Wever L, Claus F, Oyen R, Kuypers D, Evenepoel P, Bammens B, Sprangers B, Meijers B, Pirenne J, Monbaliu D, de Jonge $H$, Metalidis C, De Vusser K, Vanrenterghem Y. Intrarenal resistive index after renal transplantation. N Engl J Med. 2013;369:1797-806.

14. Wybraniec MT, Bożentowicz-Wikarek M, Chudek J, Mizia-Stec K. Preprocedural renal resistive index accurately predicts contrast-induced acute kidney injury in patients with preserved renal function submitted to coronary angiography. Int J Cardiovasc Imaging. 2017;33:595-604.

15. Schnell D, Deruddre S, Harrois A, Pottecher J, Cosson C, Adoui N, Benhamou D, Vicaut E, Azoulay E, Duranteau J. Renal resistive index better predicts the occurrence of acute kidney injury than cystatin C. Shock. 2012;38:592-7.

16. Ninet S, Schnell D, Dewitte A, Zeni F, Meziani F, Darmon M. Doppler-based renal resistive index for prediction of renal dysfunction reversibility: A systematic review and meta-analysis. J Crit Care. 2015;30:629-35.

17. Pearce JD, Craven TE, Edwards MS, Corriere MA, Crutchley TA, Fleming SH, Hansen KJ. Associations between renal duplex parameters and adverse cardiovascular events in the elderly: a prospective cohort study. Am J Kidney Dis. 2010;55:281-90.

18. Doi Y, Iwashima Y, Yoshihara F, Kamide K, Hayashi S, Kubota Y, Nakamura S, Horio T, Kawano Y. Renal resistive index and cardiovascular and renal outcomes in essential hypertension. Hypertension. 2012;60:770-7.

19. Toledo C, Thomas G, Schold JD, Arrigain S, Gornik HL, Nally JV, Navaneethan SD. Renal resistive index and mortality in chronic kidney disease. Hypertension. 2015;66:382-8.

20. Authors/Task Force members, Windecker S, Kolh P, Alfonso F, Collet JP, Cremer J, Falk V, Filippatos G, Hamm C, Head SJ, Jüni P, Kappetein AP, Kastrati A, Knuuti J, Landmesser U, Laufer G, Neumann FJ, Richter DJ, Schauerte P, Sousa Uva M, Stefanini GG, Taggart DP, Torracca L, Valgimigli M, Wijns W, Witkowski A. 2014 ESC/EACTS guidelines on myocardial revascularization: the Task Force on myocardial revascularization of the European Society of Cardiology (ESC) and the European Association for Cardio-Thoracic Surgery (EACTS)developed with the special contribution of the European Association of Percutaneous Cardiovascularlnterventions (EAPCI). Eur Heart J. 2014;35:2541-619.

21. Prejbisz A, Warchoł-Celińska E, Florczak E, Dobrowolski P, Klisiewicz A, SzwenchPietrasz E, Michałowska I, Janaszek-Sitkowska H, Kabat M, Imiela J, Januszewicz A, Januszewicz M. Renal resistive index in patients with true resistant hypertension: results from the RESIST-POL study. Kardiol Pol. 2016;74:142-50.

22. Bruno RM, Salvati A, Barzacchi M, Raimo K, Taddei S, Ghiadoni L, Solini A. Predictive value of dynamic renal resistive index (DRIN) for renal outcome in type 2 diabetes and essential hypertension: a prospective study. Cardiovasc Diabetol. 2015;14:63.

23. Geraci G, Mulè G, Mogavero M, Geraci C, D'lgnoti D, Guglielmo C, Cottone S. Renal haemodynamics and severity of carotid atherosclerosis in hypertensive patients with and without impaired renal function. Nutr Metab Cardiovasc Dis. 2015;25:160-6.

24. Geraci G, Buccheri D, Zanoli L, Fatuzzo P, Di Natale K, Zammuto MM, Nardi E, Geraci C, Mancia E, Zambelli G, Piraino D, Signorelli SS, Granata A, Cottone S, Mule G. Renal haemodynamics and coronary atherosclerotic burden are associated in patients with hypertension and mild coronary artery disease. Exp Ther Med. 2019;17:3255-63.

25. Lerolle N, Guérot E, Faisy C, Bornstain C, Diehl J-L, Fagon J-Y. Renal failure in septic shock: predictive value of Doppler-based renal arterial resistive index. Intensive Care Med. 2006;32:1553-9.

\section{Publisher's Note}

Springer Nature remains neutral with regard to jurisdictional claims in published maps and institutional affiliations. 